\author{
Marquette University \\ e-Publications@Marquette
}

$6-2000$

\title{
Reversible Carbon Monoxide Binding and Inhibition at the Active Site of the Fe-Only Hydrogenase
}

Brian Bennett

Marquette University, brian.bennett@marquette.edu

Brian J. Lemon

Utah State University

John W. Peters

Utah State University

Follow this and additional works at: https://epublications.marquette.edu/physics_fac

Part of the Physics Commons

\section{Recommended Citation}

Bennett, Brian; Lemon, Brian J.; and Peters, John W., "Reversible Carbon Monoxide Binding and Inhibition at the Active Site of the Fe-Only Hydrogenase" (2000). Physics Faculty Research and Publications. 42.

https://epublications.marquette.edu/physics_fac/42 
Marquette University

e-Publications@Marquette

\section{Physics Faculty Research and Publications/College of Arts and Sciences}

This paper is NOT THE PUBLISHED VERSION; but the author's final, peer-reviewed manuscript. The published version may be accessed by following the link in the citation below.

Biochemistry, Vol. 39, No. 25 (1 June 2000): 7455-7460. DOI. This article is @ American Chemical Society Publications and permission has been granted for this version to appear in $\underline{\mathrm{e}}-$ Publications@Marquette. American Chemical Society Publications does not grant permission for this article to be further copied/distributed or hosted elsewhere without the express permission from American Chemical Society Publications.

\section{Reversible Carbon Monoxide Binding and Inhibition at the Active Site of the Fe-Only Hydrogenase}

Brian Bennett

CCLRC Daresbury Laboratory, Warrington WA4 4AD, U.K.

Brian J. Lemon

Department of Chemistry and Biochemistry, Utah State University, Logan, Utah John W. Peters

Department of Chemistry and Biochemistry, Utah State University, Logan, Utah

\section{SUBJECTS:}

Oxides, Peptides and proteins 


\begin{abstract}
Carbon monoxide binding and inhibition have been investigated by electron paramagnetic resonance (EPR) spectroscopy in solution and in crystals of structurally described states of the Fe-only hydrogenase (Cpl) from Clostridiumpasteurianum. Simulation of the EPR spectrum of the as-isolated state indicates that the main component of the EPR spectrum consists of the oxidized state of the "H cluster" and components due to reduced accessory FeS clusters. Addition of carbon monoxide to $\mathrm{Cpl}$ in the presence of dithionite results in the inhibition of hydrogen evolution activity, and a characteristic axial EPR signal $\left[g_{\mathrm{eff}(1)}, g_{\mathrm{eff}(2)}\right.$, and $g_{\mathrm{eff}(3)}=2.0725$, 2.0061, and 2.0061, respectively] was observed. Hydrogen evolution activity was restored by successive sparging with hydrogen and argon and resulted in samples that exhibited the native oxidized EPR signature that could be converted to the reduced form upon addition of sodium dithionite and hydrogen. To examine the relationship between the spectroscopically defined states of $\mathrm{Cpl}$ and those observed structurally by X-ray crystallography, we have examined the Cpl crystals using EPR spectroscopy. EPR spectra of the crystals in the CO-bound state exhibit the previously described axial signal associated with $\mathrm{CO}$ binding. The results indicate that the addition of carbon monoxide to $\mathrm{Cpl}$ results in a single reversible carbon monoxide-bound species characterized by loss of enzyme activity and the distinctive axial EPR signal.
\end{abstract}

Metal-containing hydrogenases occur mainly in prokaryotic organisms and catalyze the reversible conversion of molecular hydrogen to protons and electrons. These enzymes function in fermentative microorganisms to regenerate oxidized electron carriers, and this is coupled to the utilization of molecular hydrogen as a source of reducing equivalents in the hydrogen oxidizing bacteria $(1,2)$. The metal-containing hydrogenases include those that contain both $\mathrm{Ni}$ and $\mathrm{Fe}$ and those that contain Fe only. Of these two forms, the NiFe hydrogenases are most often associated with hydrogen oxidation, whereas the Fe-only enzymes are most often associated with proton reduction $(1,2)$. Fourier transform infrared (FT-IR) spectroscopic studies have indicated that the active site metal-containing prosthetic groups of both classes of hydrogenases are uniquely organometallic with both metal carbonyl and metal cyanide constituents (3-5). Additionally, the structures of both classes have been determined by X-ray crystallography, revealing a unique architecture and offering insights into potential mechanisms of reversible hydrogen oxidation catalyzed by these enzymes (6-9).

The structure of the Clostridiumpasteurianum Fe-only hydrogenase (Cpl) has been determined by X-ray diffraction methods. Cpl contains $20 \mathrm{Fe}$ atoms arranged into five [Fe-S] clusters. These clusters include the enzyme active site cluster or "H cluster" and four additional [Fe-S] clusters that presumably participate in electron transfer between external electron donors and acceptors and the active site. The active site cluster comprises a regular [4Fe-4S] cubane bridged to a novel two-Fe cluster through a common cysteine sulfur (Figure 1). The two-Fe cluster is coordinated by, in addition to the cysteine sulfur, two additional sulfur atoms, five diatomic ligands (both carbon monoxide and cyanide), and a terminally bound water molecule (8). The assignment of carbon monoxide and cyanide as ligands to the active site Fe atoms is based on the results of FTIR spectroscopic studies $(3,4)$.

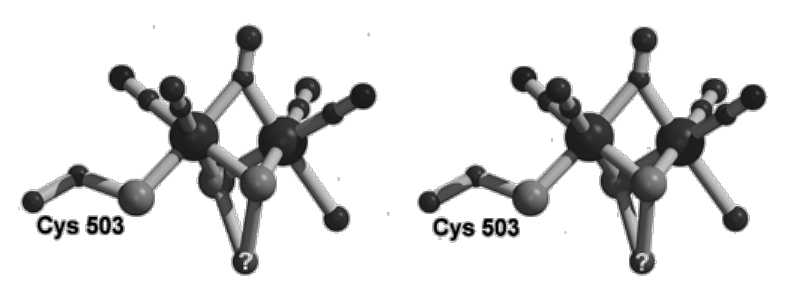

Figure 1 Wall-eyed stereoview of the two-Fe subcluster of the $\mathrm{Cpl} \mathrm{H}$ cluster. Gray scale model with Fe atoms represented as the largest dark gray atoms, $\mathrm{S}$ atoms intermediate in size and lighter gray, oxygen atoms smaller 
and lightest in color, and carbon atoms black and smallest in size. The $\mathrm{S}$ bridging moiety of unknown composition is indicated by a question mark.

The inhibition of the bidirectional Fe-only hydrogenase ( $\mathrm{Cpl}$ ) has been studied extensively, and the results have been used to support proposals regarding the mechanism of the enzyme (10-13). The first reports concerning carbon monoxide inhibition suggested that inhibition of hydrogen oxidation by carbon monoxide occurred in a competitive manner $(10,11)$. Furthermore, the observed inhibition could be reversed by subsequent photoactivation (10). More recently, however, the results of EPR studies have been interpreted to indicate that carbon monoxide binding at the active site occurs sequentially in both noninhibitory and inhibitory modes with inhibition occurring in a manner that was irreversible (12). Thus, photoactivation of carbon monoxide-treated enzyme was reinterpreted as being due to the removal of a molecule of carbon monoxide that was bound at the active site but not inhibitory (13).

The structure of $\mathrm{Cpl}$ with exogenously added carbon monoxide bound at the active site has been determined, revealing that a single molecule of carbon monoxide was bound to the active site "H cluster" at a site previously suggested as a possible site of Fe hydride and subsequent hydrogen formation (14). In these studies, binding of this single molecule of carbon monoxide resulted in the apparent complete inhibition of the enzyme. This result could therefore not be explained in light of the previous observations suggesting that during carbon monoxide inhibition two molecules of exogenously added carbon monoxide are bound at the active site $(1,12)$. At this point, a complete spectroscopic analysis of the protein crystals had not been performed and a scenario in which the previously characterized native (as crystallized) Cpl had already serendipitously bound a single molecule of carbon monoxide could not be ruled out.

Uncertainties have arisen from the apparent differences between the earlier work, describing the effects of carbon monoxide and inhibition, and the more recent work, describing the X-ray crystallographic structure of the apparent carbon monoxide-inhibited form. In the study presented here, carbon monoxide binding and inhibition of $\mathrm{Cpl}$ have been revisited by detailed EPR spectroscopic examination of $\mathrm{Cpl}$ in solution and in the crystalline state.

\section{Materials and Methods}

\section{EnzymePreparation.}

The Fe-only hydrogenase (Cpl) from C.pasteurianum was purified as described previously $(15,16)$. The carbon monoxide-inhibited form of the enzyme was generated by adding carbon monoxide (UHP grade $99.99 \%$, Matheson) to a $40.5 \mathrm{kPa}(0.4 \mathrm{~atm})$ partial pressure of $\mathrm{CO}$ with a balance of $\mathrm{Ar}$ in a sealed serum vial containing $\mathrm{Cpl}$ at a concentration of $52 \mathrm{mg} / \mathrm{mL}$ in the presence of $2 \mathrm{mM}$ sodium dithionite followed by incubation at room temperature for $5 \mathrm{~min}$. Inhibition was assessed in these samples by monitoring hydrogen evolution upon the addition of carbon monoxide in the bulk protein samples prepared for spectroscopic analysis. Hydrogen evolution activity was determined by assessing hydrogen formation with methyl viologen present as the electron carrier and sodium dithionite present as the electron donor as previously described (16). Crystallization of the native and carbon monoxide-inhibited forms of the enzyme was accomplished using the microcapillary batch diffusion method as described previously (14). For EPR investigations, $250 \mu \mathrm{L}$ samples of $0.5 \mathrm{mM} \mathrm{Cpl} \mathrm{in} 50$ $\mathrm{mM}$ Tris buffer ( $\mathrm{pH}$ 7.4) containing $200 \mathrm{mM} \mathrm{KCl}$ were used except where stated. "Oxidized" Cpl was prepared by anaerobic direct addition of 3,7-diamino-5-phenothiazinium (thionin, >85\%; Sigma) to a final concentration of $0.6 \mathrm{mM}$ (12). "Reduced" Cpl was prepared by anaerobic direct addition of sodium dithionite to a final concentration of $5 \mathrm{mM}$ (12). Incubation of samples with either $\mathrm{CO}$ or $\mathrm{H}_{2}$ was effected by agitating samples under a $9 \mathrm{~mL}$ headspace of the relevant gas at a pressure of $40.5 \mathrm{kPa}(0.4 \mathrm{~atm})$ with a balance of $\mathrm{Ar}$ at $23^{\circ} \mathrm{C}$ in sealed vials (Wheaton). Samples were transferred into EPR tubes under $\mathrm{Ar}$ and frozen immediately in liquid nitrogen. 
When crystalline samples were studied, about 20 crystals were carefully washed in a mother liquor of $100 \mathrm{mM}$ sodium acetate ( $\mathrm{pH} 4.6$ ) containing $25 \% \mathrm{w} / \mathrm{v}$ poly(ethylene glycol) 4000 and $200 \mathrm{mM}$ ammonium acetate and resuspended in $50 \mu \mathrm{L}$ of the same liquor prior to anaerobic transfer to quartz EPR tubes (Wilmad) and immediate freezing in liquid nitrogen.

\section{EPRSpectroscopy.}

EPR spectra were recorded on a Bruker ESP 300E spectrometer equipped with an Oxford Instruments ESR-900 helium flow cryostat and an ER 4116 DM dual mode X-band cavity operating at 9.64-9.65 GHz. Precise microwave frequencies were recorded for each spectrum, and all spectra are presented aligned with a magnetic field range corresponding to a microwave frequency of $9.648000 \mathrm{GHz}$. A magnetic field modulation frequency of $100 \mathrm{kHz}$ was employed throughout. Other EPR operating parameters are specified in the figure legends. Residual signals due to cavity impurities were removed as in earlier work (17-19). EPR simulations were performed as described previously (20). Where spectra were due to more than one signal, as indicated by simulation, relative amounts of the contributing signals were estimated by double integration of simulations of the individual components. Overall spin quantitation of experimental spectra was performed by comparing integrations with a $2 \mathrm{mM} \mathrm{Cu}^{2+}$-EDTA standard with corrections applied for transition probabilities, temperature, and microwave power as described elsewhere (21). For estimation of the "CO-treated" signal, correction factors for temperature dependence were estimated from the data of Adams (12).

\section{Results and Discussion}

\section{OxidizedCpl.}

Oxidized $\mathrm{Cpl}$ exhibited a characteristic rhombic EPR signal due to a single paramagnetic species (Figure 2a). The signal is due to the oxidized $\mathrm{H}$ cluster which exhibits an $S=1 / 2$ paramagnetic ground state, and simulation of this species (Figure 2b) provided the EPR parameters $g_{\mathrm{eff(1)}}, g_{\mathrm{eff}(2)}$, and $g_{\mathrm{eff}(3)}=2.0973,2.0392$, and 1.9993, respectively, in good agreement with the previously reported $g$ values for this signal (12). Upon addition of $\mathrm{CO}$, the rhombic signal was replaced by an axial signal, shown as Figure $2 \mathrm{c}$, which was simulated (Figure $2 \mathrm{~d}$ ) assuming $g_{\mathrm{eff}(1)}, g_{\mathrm{eff}(2)}$, and $g_{\mathrm{eff}(3)}=2.0725,2.0061$, and 2.0061, respectively. This species was then flushed with argon for 15 and $60 \mathrm{~min}$, and the EPR spectra were recorded and shown as traces e and $\mathrm{g}$ of Figure 2, respectively. Simulation of the spectrum exhibited by the sample after flushing with Ar for 15 min (Figure 2f) indicated that the spectrum consists of a $96 \%$ contribution due to the signal from the oxidized enzyme and only a $4 \%$ contribution due to the "CO species". After $60 \mathrm{~min}$, the spectrum had reverted entirely to the oxidized species, indicating the binding of $\mathrm{CO}$ to oxidized $\mathrm{Cpl}$ is completely reversible. 


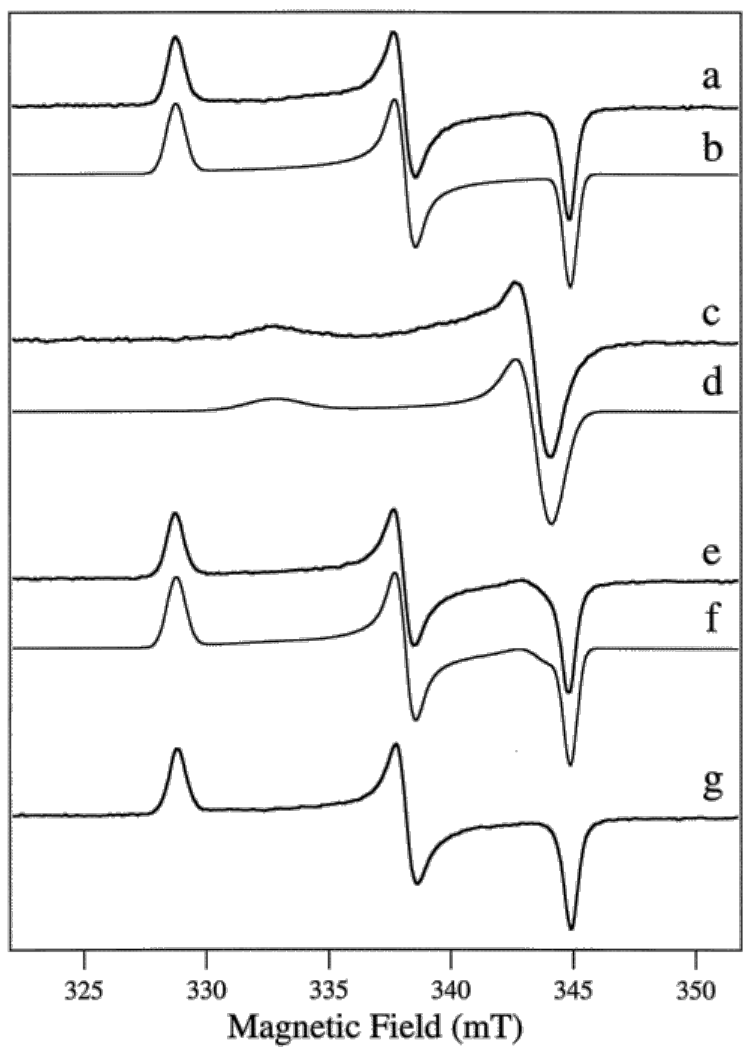

Figure 2 EPR spectra from oxidized and CO-treated Cpl. The traces show EPR signals from a single $0.5 \mathrm{mM}$ sample of $\mathrm{Cpl}$ in $50 \mathrm{mM}$ Tris buffer (pH 7.4) containing $200 \mathrm{mM} \mathrm{KCl}$ after sequential treatments as follows: (a) anoxic oxidation with $0.6 \mathrm{mM}$ thionin, (c) anaerobic exposure to $40.5 \mathrm{kPa}(0.4 \mathrm{~atm}) \mathrm{CO}$ for $5 \mathrm{~min}$, (e) anaerobic flushing with Ar for $15 \mathrm{~min}$, and (g) anaerobic flushing with Ar for $60 \mathrm{~min}$. Traces $b$, d, and $\mathrm{f}$ are computer simulations of traces a, c, and g, respectively, assuming for trace a, $g_{\mathrm{eff}(1)}, g_{\mathrm{eff}(2)}$, and $g_{\mathrm{eff}(3)}=2.0973,2.0392$, and 1.9993, respectively; for trace d, $g_{\text {eff(1) }}, g_{\text {eff(2) }}$, and $g_{\text {eff(3) }}=2.0725,2.0061$, and 2.0061, respectively; and for trace f, there is a $4.4 \%$ contribution from trace $d$ and a $95.6 \%$ contribution from trace b. Spectra were recorded at 15 (a,

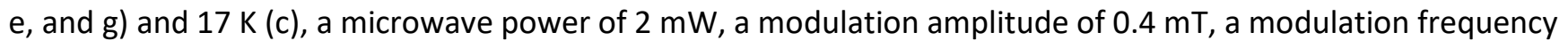
of $100 \mathrm{kHz}$, and microwave frequencies of $9.64-9.65 \mathrm{GHz}$; precise microwave frequencies were recorded, and spectra are presented aligned with a magnetic field range corresponding to a microwave frequency of 9.648000 $\mathrm{GHz}$. Spectra are presented normalized for peak-to-baseline intensities. Fractional contributions to trace $f$ were calculated by double integration of the individual components that were summed to provide the simulation.

\section{As-IsolatedandReducedCpl.}

The EPR spectrum of as-isolated $\mathrm{Cpl}$ at $12 \mathrm{~K}$ is presented as Figure $3 \mathrm{a}$ and is consistent with the spectra for asisolated Cpl reported in the early 1970s (22). The spectrum is complex and clearly consists of more than one paramagnetic species. The spectrum appears to consist of a number of well-defined, and therefore magnetically isolated, $S=1 / 2$ species and of a broader ill-defined signal, perhaps due to magnetically coupled centers. The contribution to the spectrum due to magnetically isolated reduced [Fe-S] clusters could be simulated (Figure 3b) by assuming three species, one of which corresponded to the oxidized $\mathrm{H}$ cluster (Figure 3c), a second species

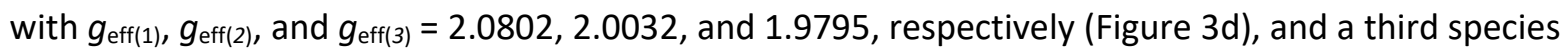

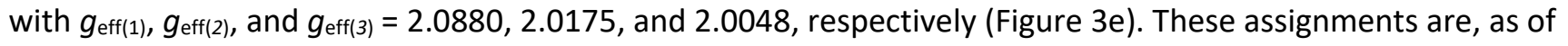
yet, tentative, and further detailed characterization of the relaxation properties of these signals is in progress to facilitate unambiguous characterization of the signals and assignment to specific crystallographically identified [Fe-S] clusters. Careful examination of the broad underlying feature in the spectrum revealed a close similarity to the spectrum of dithionite-reduced Cpl (Figure 3f). This spectrum is ill-defined and very broad and is 
characteristic of extensively spin-coupled multiple iron-sulfur clusters. Inclusion of a proportion of this signal corresponding to $0.9 \mathrm{spin} \mathrm{mol}^{-1}$ further improved the simulation (Figure $3 \mathrm{~g}$ ) of the experimental spectrum.

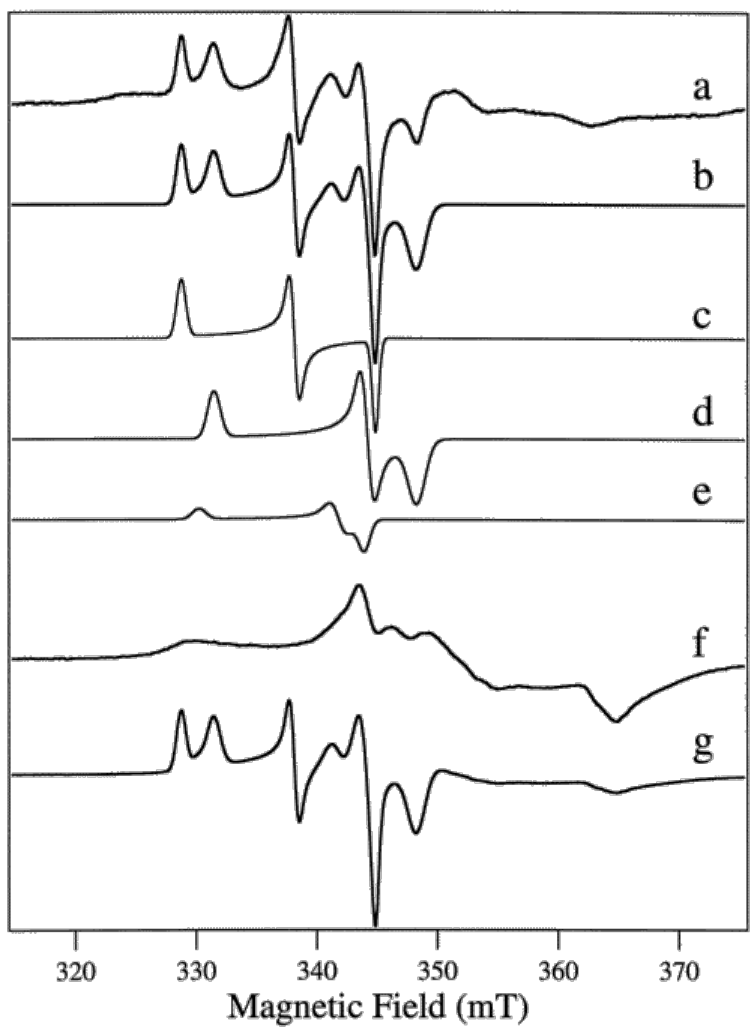

Figure 3 EPR signal from as-prepared Cpl. Trace a shows the EPR signal from as-prepared $\mathrm{Cpl}$ in $50 \mathrm{mM}$ Tris buffer ( $\mathrm{pH} 7.4$ ) containing $200 \mathrm{mM} \mathrm{KCl}$. Trace $b$ is a computer simulation of trace a assuming three distinct isolated species (c-e), with fractional contributions of (c) $38.0 \%$, (d) $51.4 \%$, and (e) $10.6 \%$. Traces c-e are the

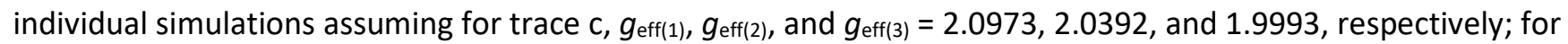
trace d, $g_{\mathrm{eff}(1)}, g_{\mathrm{eff}(2)}$, and $g_{\mathrm{eff}(3)}=2.0802,2.0032$, and 1.9795, respectively; and for trace e, $g_{\mathrm{eff}(1),} g_{\mathrm{eff}(2)}$, and $g_{\mathrm{eff}(3)}=$ $2.0880,2.0175$, and 2.0048, respectively. Trace $\mathrm{f}$ is the EPR signal from Cpl reduced with $5 \mathrm{mM}$ sodium dithionite. Trace $g$ is an alternative simulation of trace a obtained by summing a $44.6 \%$ contribution of trace $b$ and a $55.4 \%$ contribution of trace $\mathrm{f}$. Experimental spectra were recorded at $15 \mathrm{~K}$, a microwave power of $1 \mathrm{~mW}$, a modulation amplitude of $0.4 \mathrm{mT}$ (a) or $1.0 \mathrm{mT}$ (f), a modulation frequency of $100 \mathrm{kHz}$, and microwave frequencies of 9.64-9.65 GHz; precise microwave frequencies were recorded, and spectra are presented aligned with a magnetic field range corresponding to a microwave frequency of $9.648000 \mathrm{GHz}$. Spectra are presented with arbitrary intensities. Fractional contributions to traces $b$ and $g$ were calculated by double integration of the individual components which were summed to provide the simulation.

Cpl was reduced from the as-isolated state with 10 equiv of sodium dithionite, and the EPR spectrum is presented as Figure 4c. Upon incubation of reduced $\mathrm{Cpl}$ under $\mathrm{CO}$, hydrogen evolution activity was undetectable and a new complex signal was observed (Figure 4d). This complex signal integrated to about 2 spins mol $^{-1}$ and is composed of about $50 \%$ of the $\mathrm{CO}$ spectrum observed upon incubation of oxidized $\mathrm{Cpl}$ with $\mathrm{CO}$ (cf. traces $\mathrm{c}$ and $\mathrm{d}$ of Figure 2 ) and $50 \%$ of a complex, poorly resolved spectrum. The appearance of the $\mathrm{CO}$ signal was clearly unrelated to adventitious oxidation of the enzyme by $\mathrm{O}_{2}$; not only were all experiments carried out strictly anaerobically, but the reduced enzyme was also maintained in the presence of sodium dithionite. Furthermore, at no stage was a signal indicative of $\mathrm{O}_{2}$ binding to the $\mathrm{H}$ cluster observed $(1,13)$. The broader signal upon which the CO signal is superimposed was intermediate in appearance between that of the as-prepared enzyme and the dithionite-reduced enzyme. The outermost features of the signal were broad and asymmetric, exhibiting line 
shapes that are analogous to the outer lines of the reduced signal. However, these lines are shifted toward the center of the signal relative to the reduced signal; the low-field line has moved from 329.8 to $332.9 \mathrm{mT}$, and the high-field line has moved from 364.8 to $358.6 \mathrm{mT}$.

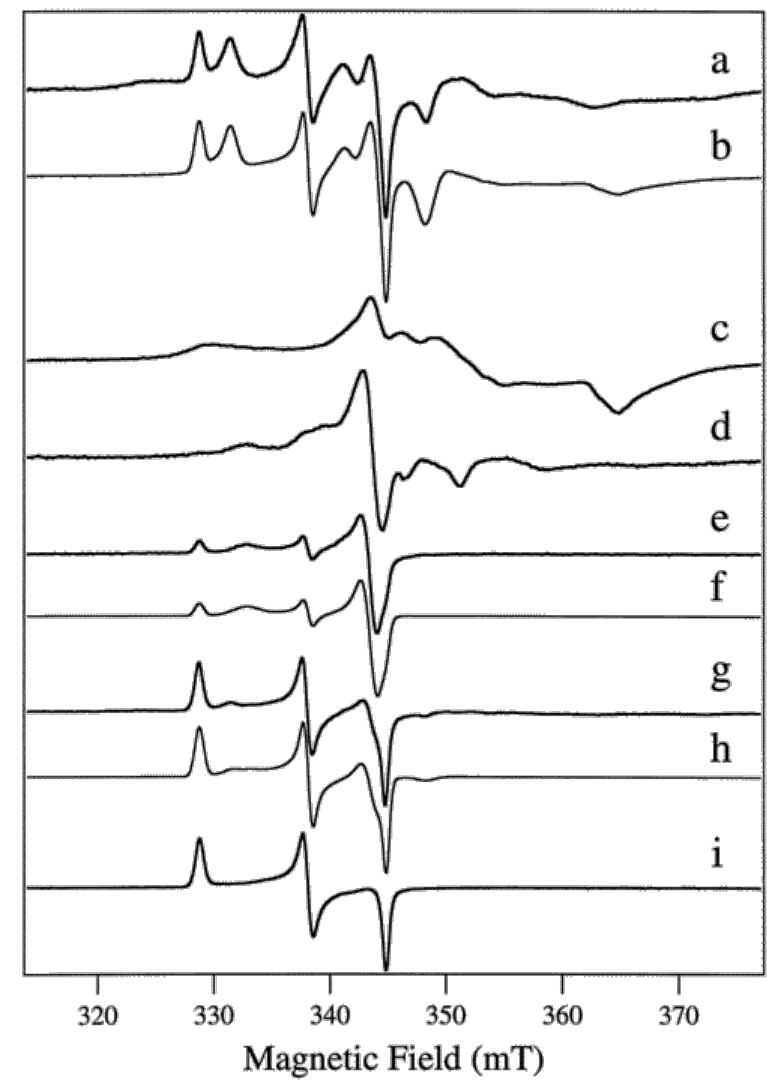

Figure 4 EPR signals from as-prepared, reduced, and CO-treated Cpl. Trace a shows the spectrum of as-prepared $\mathrm{Cpl}$ in $50 \mathrm{mM}$ Tris buffer (pH 7.4) containing $200 \mathrm{mM} \mathrm{KCl}$ (cf. Figure 3a). Trace $b$ is the simulation of trace a shown as Figure $3 \mathrm{~g}$, generated as described in the legend of Figure 3. Traces c-e, g, and i are EPR spectra of $\mathrm{Cpl}$ upon (c) reduction with hydrogen in the presence of sodium dithionite, and subsequent exposure to 0.4 atm CO for $5 \mathrm{~min}$, (d) followed by flushing anaerobically with Ar for $5 \mathrm{~min}$, (e) addition of $\mathrm{H}_{2}$ and dithionite and incubation for $3 \mathrm{~min}$ followed by flushing with Ar for additional $5 \mathrm{~min},(\mathrm{~g})$ and again incubation with $\mathrm{H}_{2}$ and 1 $\mathrm{mM}$ dithionite for $3 \mathrm{~min}$ followed by flushing with $\mathrm{Ar}$ for an additional $5 \mathrm{~min}$ (i). Traces $\mathrm{f}$ and $\mathrm{h}$ are simulations assuming mixtures of the simulations of "oxidized" and "CO-treated" Cpl shown as traces b and d of Figure 2, respectively. Trace $f$ assumes $0.29 \mathrm{spin} \mathrm{mol}^{-1}$ of the oxidized $\mathrm{Cpl}$ species and $0.71 \mathrm{spin} \mathrm{mol} \mathrm{m}^{-1}$ of the CO-treated species; trace $h$ assumes 0.79 and $0.21 \mathrm{spin} \mathrm{mol}^{-1}$ of the oxidized and CO-treated species, respectively, and a 0.05 spin mol-1 contribution from signal A. Spectra were recorded at $15 \mathrm{~K}$, a microwave power of $2 \mathrm{~mW}$, a modulation amplitude of $0.4(\mathrm{a}, \mathrm{d}, \mathrm{e}, \mathrm{g}$, and i) or $1.0 \mathrm{mT}$ (c), a modulation frequency of $100 \mathrm{kHz}$, and microwave frequencies of 9.64-9.65 GHz; precise microwave frequencies were recorded, and spectra are presented aligned with a magnetic field range corresponding to a microwave frequency of $9.648000 \mathrm{GHz}$. Spectra are presented with arbitrary intensities. Fractional contributions to traces $b$, $f$, and $h$ were calculated by double integration of the individual components that were summed to provide the simulations.

Other features in the spectrum of reduced $\mathrm{Cpl}$ upon short exposure to $\mathrm{CO}$ (Figure $4 \mathrm{~d}$ ) include resolved lines at 346.6 and $351.2 \mathrm{mT}$, which may or may not be related to the lines in the reduced spectrum at 344 and $348 \mathrm{mT}$, and a poorly resolved feature centered at $340 \mathrm{mT}$. The resolution of lines in the spectrum of CO-treated reduced $\mathrm{Cpl}$ and, particularly, the movement of the high- and low-field lines toward the center of the spectrum are indicative of a dimunition of the spin coupling observed for the iron-sulfur clusters. The most likely explanation 
for this is that one of the clusters has become oxidized and the overall magnetic interaction experienced by each of the other clusters is reduced. A very similar phenomenon has been described for the iron-sulfur clusters of Escherichiacoli membrane-bound respiratory nitrate reductases $A$ and Z. E.coli nitrate reductase contains three $[4 \mathrm{Fe}-4 \mathrm{~S}]^{2+/ 1+}$ and one $[3 \mathrm{Fe}-4 \mathrm{~S}]^{1+/ 0}$ cluster and is comparable to $\mathrm{Cpl}$ in terms of its complement of paramagnetic centers in the oxidized and reduced forms (23-25). Upon reduction of the [3Fe-4S] cluster and one [4Fe-4S] cluster, no magnetic coupling is indicated by the EPR spectrum, though the $[3 \mathrm{Fe}-4 \mathrm{~S}]^{0}$ cluster is likely in the $S=2$ state. Upon reduction of a second [4Fe-4S] cluster, a degree of magnetic interaction is observed, but upon reduction of the third, low-potential [4Fe-4S] cluster, extensive magnetic interactions are observed and a broad, essentially featureless signal is observed which is not dissimilar to that from reduced Cpl.

The EPR spectrum of CO-treated reduced $\mathrm{Cpl}$ presented in Figure $4 \mathrm{~d}$ indicates not only oxidation of the $\mathrm{H}$ cluster upon formation of the CO-bound species but also partial oxidation of the iron-sulfur complement of the enzyme. This in turn may be indicative of catalysis of slow proton reduction by $\mathrm{Cpl}$ occurring in the presence of dithionite despite the presence of $\mathrm{CO}$, suggesting that $\mathrm{CO}$ is a competitive rather than an irreversible inhibitor. This is consistent with observations concerning specific activity measurements that indicate that activity can be restored if $\mathrm{CO}$ is removed from concentrated samples. In addition, when small aliquots of concentrated COinhibited $\mathrm{Cpl}$ are transferred to assay vials that do not contain $\mathrm{CO}$, full activity [ $3100 \mathrm{nmol}$ of $\mathrm{H}_{2}$ formed min ${ }^{1}$ (mg of Cpl) ${ }^{-1}$ ] is observed.

Following incubation of reduced $\mathrm{Cpl}$ under $\mathrm{CO}$ for $5 \mathrm{~min}$, the sample was flushed with Ar gas for 5 min and the EPR signal recorded (Figure 4e). An excellent simulation was obtained (Figure 4f) which assumed $0.18 \mathrm{spin} \mathrm{mol}^{-}$ ${ }^{1}$ due to the oxidized signal and $0.82 \mathrm{spin} \mathrm{mol}^{-1}$ for the $\mathrm{CO}$ species. No signals due to iron-sulfur clusters were detected, indicative of complete oxidation of the iron-sulfur complement. The Cpl sample atmosphere was then exchanged with $100 \% \mathrm{H}_{2}$, and $1 \mathrm{mM}$ dithionite was added. After a 3 min incubation period under $\mathrm{H}_{2}$, the sample was flushed with Ar as before, yielding a spectrum (Figure $4 \mathrm{~g}$ ), of which simulation (Figure $4 \mathrm{~h}$ ) indicated that only $21 \%$ of the $\mathrm{H}$ clusters in the sample were now in the CO-bound form, with the remainder exhibiting the oxidized signal. The addition of $\mathrm{H}_{2}$ to the $\mathrm{CO}$-bound and inhibited $\mathrm{Cpl}$ stimulated the removal of $\mathrm{CO}$, consistent with the previously observed competitive manner of inhibition with respect to hydrogen oxidation $(10,11)$. After an additional cycle of $\mathrm{H}_{2}$ incubation ( $3 \mathrm{~min}$ ) and Ar flushing, the resulting spectrum was completely due to the oxidized $\mathrm{H}$ cluster (Figure 4i). Complete removal of $\mathrm{CO}$ could be accomplished with samples without addition of $\mathrm{H}_{2}$ by multiple cycles of Ar flushing over a period of $1 \mathrm{~h}$.

Upon incubation of the reduced $\mathrm{Cpl}$ with $\mathrm{CO}$, a species is generated that is indistinguishable from the $\mathrm{CO}$-bound $\mathrm{H}$ cluster of the oxidized enzyme. $\mathrm{CO}$ is not redox active, and therefore, oxidation of both the $\mathrm{H}$ cluster and the iron-sulfur clusters can only arise as a consequence of proton reduction by the reduced enzyme. The EPR data indicate that this process can occur slowly in the presence of $\mathrm{CO}$ and that $\mathrm{CO}$ cannot be an irreversibly binding inhibitor of either the oxidized or reduced enzyme. Irreversible binding of $\mathrm{CO}$ to the reduced $\mathrm{H}$ cluster would result in an EPR-silent species that would be unable to reduce protons and therefore would remain in the reduced state. However, estimations of the $\mathrm{CO}$ signal's contribution to Figure $4 \mathrm{e}$ by integration and to Figure 4d by observation of 0.71 and about 1 spin $\mathrm{mol}^{-1}$, respectively, clearly suggest that prior to CO removal most of the $\mathrm{H}$ clusters in a frozen sample are trapped in the oxidized CO-bound form. Yet, the lack of an iron-sulfur component in the spectrum of Figure $4 \mathrm{e}$ compared to Figure $4 \mathrm{~d}$ clearly shows that the enzyme is still capable of redox reaction even though the $\mathrm{H}$ clusters are largely in the $\mathrm{CO}$-bound form. The only simple scenario consistent with all the data, then, is that $\mathrm{CO}$ is a reversible, competitive inhibitor of $\mathrm{Cpl}$. It is entirely possible that $\mathrm{CO}$ does not bind the reduced $\mathrm{H}$ cluster of $\mathrm{Cpl}$ at all or else is highly labile. Indeed, demonstration of $\mathrm{CO}$ binding to the reduced cluster would be very difficult. The putative $\mathrm{CO}$-bound reduced $\mathrm{H}$ cluster would likely be EPRspectroscopically silent. The reduced $\mathrm{H}$ cluster in the presence of $\mathrm{CO}$ is unstable in that it reverts to the oxidized CO-bound form, presumably due to slow turnover, so radioactive tracing with ${ }^{14} \mathrm{CO}$ is not practical. Vibrational 
spectroscopic methods would have to be carried out in the frozen state and would be hindered by the presence of Fe-CO moieties in the resting enzyme. Evaluation of the practicability of rapid diffusion of $\mathrm{CO}$ into reduced crystals of $\mathrm{Cpl}$ followed by flash-freezing is ongoing. Regardless, binding of $\mathrm{CO}$ to the oxidized cluster clearly does occur, though the Ar flushing experiments suggest a bulk time constant for dissociation of $\mathrm{CO}$ of around 2-7 min, depending on precise conditions.

\section{CrystallineCpl.}

The data from solution studies with $\mathrm{Cpl}$ clearly indicate that $\mathrm{CO}$ binds reversibly to the enzyme, yielding a single, characteristic EPR-detectable species of the $\mathrm{H}$ cluster. Structural characterization of the CO-inhibited form of $\mathrm{CpI}$ (14) indicated that, in addition to the ligands to the terminal iron atom found in the native Cpl structure, Fe2 (Figure 1), electron density consistent with the presence of an additional single CO molecule bound to Fe2. However, reports in the literature (13) have suggested that the EPR signal is, in fact, due to binding of a CO molecule that is not inhibitory. In the complex scenario previously proposed, both a noninhibitory CO molecule and a second irreversibly binding inhibitory molecule will react with the enzyme under conditions of enzyme turnover. We therefore wished to investigate whether the crystallographically characterized Cpl species, in which only one molecule of exogenous $\mathrm{CO}$ is observed bound, corresponded to the $\mathrm{CO}$ species observed by EPR.

An EPR spectrum of crystals of as-isolated $\mathrm{Cpl}$ is presented as Figure $5 \mathrm{a}$. The spectrum contained features also present in the spectrum of as-isolated $\mathrm{Cpl}$ in solution (cf. Figure $5 \mathrm{~b}$ ). Features identifiable as being due to the oxidized signal were observed at 329 and $337 \mathrm{mT}$, and a split feature was observed at $344 \mathrm{mT}$. Some of these features were shifted slightly from the corresponding positions in the spectrum of the enzyme in frozen solution. Indeed, the spectrum exhibited some sensitivity to the orientation of the sample in the spectrometer and the precise positions of the features shifted around the positions observed in the solution spectra as a function of sample orientation. Approximately 20 crystals were washed and suspended in synthetic mother liquor prior to transfer to an EPR tube, and it is likely that 10-15 crystals were in the frozen drop that was positioned between the cavity and the modulation coils. Therefore, a true "powder spectrum" could not be obtained, and the observed spectrum represents the sum of 10-15 single-crystal spectra, each of which has resonance positions dependent upon the precise orientation of the crystal. Nevertheless, the approximation to a powder spectrum is sufficient to allow identification of the oxidized $\mathrm{H}$ cluster signal.

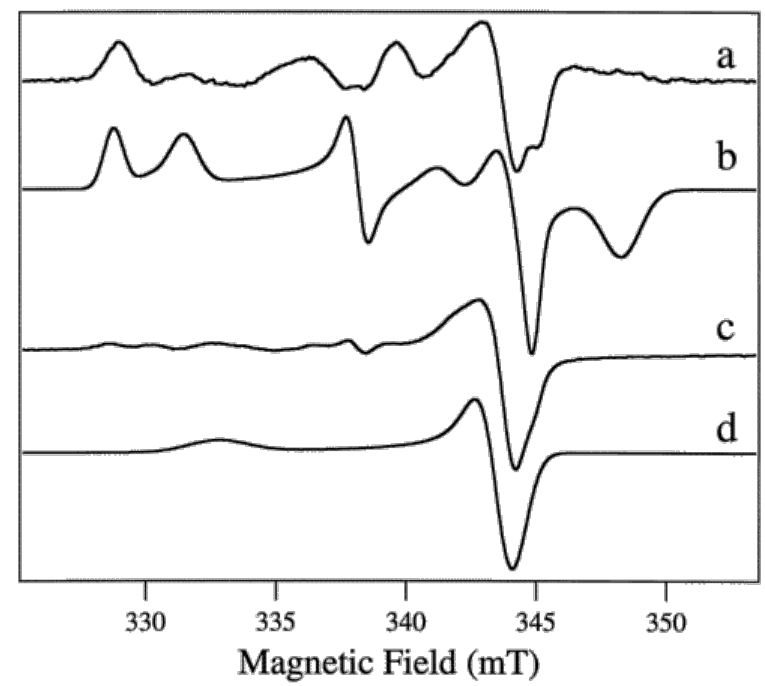

Figure 5 EPR signals from crystalline Cpl. EPR spectra are presented of crystals of (a) as-prepared and (c) COtreated Cpl, in $100 \mathrm{mM}$ sodium acetate (pH 4.6) containing 25\% w/v poly(ethylene glycol) 4000 and $200 \mathrm{mM}$ ammonium acetate. About 20 crystals were maintained in suspension in crystallization mother liquor and frozen rapidly prior to EPR spectroscopic investigation. For comparison, simulations of frozen solution spectra of asprepared and CO-treated $\mathrm{Cpl}$ in $50 \mathrm{mM}$ Tris buffer (pH 7.4) containing $200 \mathrm{mM} \mathrm{KCl}$ are shown as traces b and d, 
respectively (also shown and described as Figures $3 \mathrm{~b}$ and $2 \mathrm{~d}$ ). Experimental spectra were recorded at 15 (a) and $12 \mathrm{~K}$ (c), a microwave power of $2 \mathrm{~mW}$, a modulation amplitude of $0.4 \mathrm{mT}$, and a modulation frequency of 100 $\mathrm{kHz}$ and are presented aligned with a magnetic field range corresponding to a microwave frequency of 9.648000 $\mathrm{GHz}$.

EPR analysis of crystals of the CO bound state of Cpl yielded a spectrum (Figure $5 c$ ) that is almost entirely (>95\%) due to a $\mathrm{CO}$ signal that is indistinguishable from that observed in frozen solution. Therefore, it can be concluded that the species characterized by EPR and that characterized by crystallography are the same and that the crystallographically characterized species corresponds to an inhibited species of $\mathrm{Cpl}$ containing a single molecule of $\mathrm{CO}$ reversibly bound to the $\mathrm{H}$ cluster.

\section{Acknowledgment}

We thank Dr. Richard Holz, Dr. Jennifer Huyett, and Dr. Se Bok Jang for assistance and discussions.

\section{References}

1 Adams, M. W. W. (1990) Biochim.Biophys.Acta1020, 115-45.

2 Przybyla, A. E., Robbins, J., Menon, N., and Peck, H. D., Jr. (1992) FEMSMicrobiol. Rev.8, 109-35.

3 Happe, R. P., Roseboom, W., Pierik, A. J., Albracht, S. P., and Bagley, K. A. (1997) Nature385, 126.

4 Pierik, A. J., Hulstein, M., Hagen, W. R., and Albracht, S. P. (1998) Eur. J.Biochem. 258, 572-8.

5 van der Spek, T. M., Arendsen, A. F., Happe, R. P., Yun, S., Bagley, K. A., Stufkens, D. J., Hagen, W. R., and Albracht, S. P. (1996) Eur. J.Biochem.237, 629-34.

6 Volbeda, A., Charon, M. H., Piras, C., Hatchikian, E. C., Frey, M., and Fontecilla-Camps, J. C. (1995) Nature373, $580-7$.

7 Higuchi, Y., Yagi, T., and Yasuoka, N. (1997) Structure5, 1671-80.

8 Peters, J. W., Lanzilotta, W. N., Lemon, B. J., and Seefeldt, L. C. (1998) Science282, 1853-8.

9 Nicolet, Y., Piras, C., Legrand, P., Hatchikian, C. E., and Fontecilla-Camps, J. C. (1999) Structure7, 13-23.

10 Thauer, R. K., Kaufer, B., Zahringer, M., and Jungermann, K. (1974) Eur.J.Biochem. 42, 447-52.

11 Erbes, D. L., and Burris, R. H. (1978) Biochim. Biophys.Acta525, 45-54.

12 Adams, M. W. (1987) J.Biol.Chem.262, 15054-61.

13 Kowal, A. T., Adams, M. W., and Johnson, M. K. (1989) J.Biol.Chem.264, 4342-8.

14 Lemon, B. J., and Peters, J. W. (1999) Biochemistry38, 12969-73.

15 Chen, J. S., and Mortenson, L. E. (1974) Biochim.Biophys.Acta371, 283-98.

16 Adams, M. W. W., and Mortenson, L. E. (1984) Biochim. Biophys.Acta766, 51-61.

17 Bennett, B., and Holz, R. C. (1997) Biochemistry36, 9837-46.

18 Bennett, B., and Holz, R. C. (1998) J.Am.Chem.Soc.120, 1923-33.

19 Bennett, B., and Holz, R. C. (1998) J.Am.Chem.Soc.120, 12139-40.

20 Butler, C. S., Charnock, J. M., Bennett, B., Sears, H. J., Reilly, A. J., Ferguson, S. J., Garner, C. D., Lowe, D. J., Thomson, D. J., Berks, B. C., and Richardson, D. J. (1999) Biochemistry38, 9000-12.

21 Barber, M. J., Bray, R. C., Lowe, D. J., and Coughlan, M. P. (1976) Biochem.J.153, 297-307.

22 Nakos, G., and Mortenson, L. E. (1971) Biochemistry10, 2442-9.

23 Johnson, M. K., Bennett, D. E., Morningstar, J. E., Adams, M. W. W., and Mortenson, L. E. (1985) J.Am.Chem.Soc.107, 5456-63.

24 Guigliarelli, B., Asso, M., More, C., Augier, V., Blasco, F., Pommier, J., Giordano, G., and Bertrand, P. (1992) Eur.J.Biochem.207, 61-8.

25 Bennett, B., and Bray, R. C. (1994) Biochem.Soc.Trans.22, 283. 